J Neurosurg (Spine 3) 99:306-309, 2003

\title{
Upper thoracic spinal cord herniation after traumatic nerve root avulsion
}

\author{
Case report and review of the literature
}

\author{
Victor R. DaSilva, M.D., Mubarak Al-Gahtany, M.D., F.R.C.S.(C), \\ RaJiv Midha, M.D., M.Sc., F.R.C.S.(C), DiPanka SARMa, M.D., \\ AND Perry CoOPER, M.D., F.R.C.P.(C) \\ Division of Neurosurgery, Department of Surgery, and the Division of Neuroradiology, Department of \\ Radiology, Sunnybrook and Women's College Health Sciences Centre, University of Toronto, Ontario, \\ Canada
}

\begin{abstract}
$\checkmark$ Transdural herniation of the spinal cord, a rare but well-documented entity, has been reported sporadically for more than 25 years as a possible cause for various neurological signs and symptoms ranging from isolated sensory or motor findings to myelopathy and Brown-Séquard syndrome. The authors report, to the best of their knowledge, the first case of upper thoracic spinal cord herniation occurring after traumatic nerve root avulsion.
\end{abstract}

\section{KEY WORDS • Brown-Séquard syndrome • nerve root avulsion • pseudomeningocele • spinal cord herniation - spinal cord tethering}

$\mathrm{S}$ PINAL cord herniation is an uncommon entity that can occur spontaneously or after surgery or trauma. Idiopathic (spontaneous) spinal cord herniation is usually seen anteriorly and commonly in the thoracic spine. It most commonly affects middle-age women and manifests with Brown-Séquard syndrome and myelopathy without radiological evidence of compressive lesions.

Postsurgical or traumatic spinal cord herniation, on the other hand, can be found anywhere in the spinal column and can present clinically, in an acute or delayed fashion, with a much broader spectrum of signs and symptoms. ${ }^{13,16}$ We describe the case of a 41-year-old man with thoracic spinal cord herniation who presented 19 years after sustaining a traumatic brachial plexus nerve root avulsion. The pathogenesis, clinical and imaging spectra, treatment alternatives, and outcome are discussed.

\section{Case Report}

History. This 41-year-old man was involved in a motorcycle accident in 1982, 19 years prior to his presentation. At that time, he sustained a significant head injury, which rendered him comatose and required intensive care unit admission. During that time, he also suffered marked right brachial plexus and right lower leg injuries. The brachial plexus injury resulted in a flail arm; elements of the brachial plexus from $\mathrm{C}-5$ to $\mathrm{T}-1$ were involved, with the

Abbreviations used in this paper: CSF = cerebrospinal fluid; $\mathrm{CT}=$ computerized tomography; $\mathrm{MR}=$ magnetic resonance. lower nerve roots known to be avulsed. The patient's right arm was amputated 18 months later after no improvement was exhibited. Subsequently, he developed phantom pain syndrome, which was managed nonsurgically. His lower right leg required multiple reconstructive procedures and 5 years later, with a shorter leg, he was finally able to walk independently.

Two years prior to his presentation, he started to complain of new right leg weakness and a tendency to fall, which was particularly noticeable while climbing stairs. No neck, back, or leg pain was noted and he denied any sensory, bowel, or bladder dysfunction.

Examination. On examination, a right Horner syndrome was present. Amputation of his right arm at mid-level was apparent. There was marked wasting and weakness of the intrinsic muscles of his left hand. The bulk and strength of the remaining muscles of that extremity as well as the tendon reflexes were normal. He exhibited wasting of the right thigh and calf muscles, with shortening of the leg. There was mild weakness (Grade 4/5) of the right hip and knee flexors and right tibialis anterior, and Grade 3/5 of the right extensor hallucis longus. An upgoing toe and hyperreflexia of the knee and ankle jerks were also present ipsilaterally. Normal strength, tone, and bulk of left lowerextremity muscles were noted. The sensory examination to pinprick, joint position, and vibration was normal in both lower extremities and left arm.

The patient underwent MR imaging and CT myelography (Figs. 1-3). The MR imaging studies revealed a lateral pseudomeningocele arising from the right T1-2 inter- 


\section{Upper thoracic spinal cord herniation}

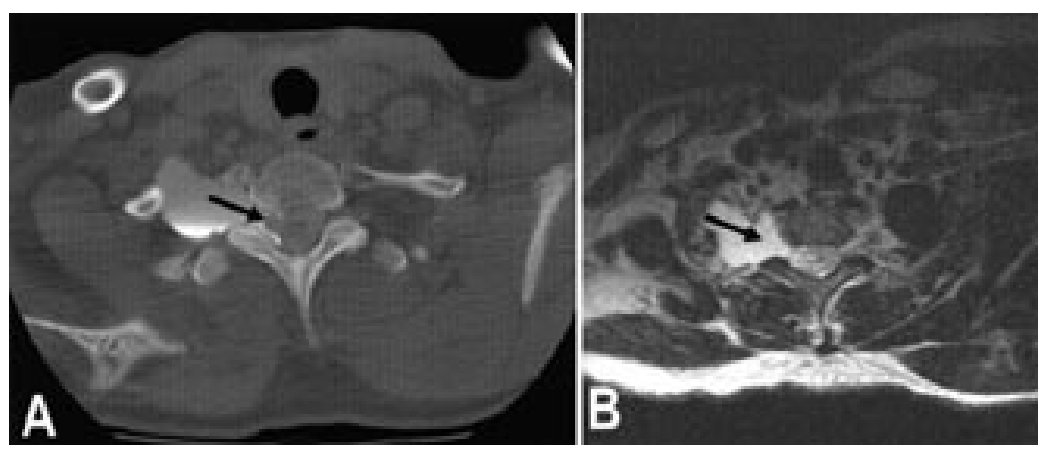

FIG. 1. A: Axial postmyelography CT scan demonstrating extravasation of contrast material into pseudomeningocele and prolapse of the spinal cord (arrow). B: Axial fast-spin echo $\mathrm{T}_{2}$-weighted MR image revealing tethering of the spinal cord and pseudomeningocele (arrow).

vetebral foramen, resting on the chest apex, with spinal cord atrophy, herniation, and tethering. Intramedullary hyperintensity was also present on fast-spin echo $\mathrm{T}_{2}$-weighted sequences. Postmyelography CT scanning demonstrated extravasation of contrast into the cystic cavity, which consisted of intra- and extraspinal portions.

Operation. Because of the patient's progressive symptoms surgery was deemed necessary. This was performed via a posterior approach. We conducted T-1 and T-2 laminectomies. Intraoperatively attenuation and outpouching of the spinal cord were seen through a 6-mm-diameter dural defect (Fig. 4). Clear communication of CSF was identified between the spinal subarachnoid space and intra- and extraspinal components of the pseudomeningocele. Arachnoidal bands tethered the spinal cord to the dural ring. Meticulous dissection and lysis of such bands allowed reduction of the herniated cord (Fig. 4). Primary closure was supplemented using a dural patch (Duragen; Integra Neurosciences, Plainsboro, NJ) to seal the track between the subarachnoid space and the pseudomeningocele (Fig. 4 ), as well as to prevent spinal cord reherniation.

Postoperative Course. Six weeks postoperatively, he exhibited a subjective improvement of the right leg weakness and experienced less difficulty when climbing stairs. Continuing improvement of his myelopathy was evident at the 6-month follow-up examination.

\section{Discussion}

Spinal cord herniation is an entity that rarely occurs, with little more than 50 cases reported and approximately 30 surgically proven cases. ${ }^{16,21,28}$ Spinal cord herniation can be classified as spontaneous, posttraumatic, and iatrogenic. ${ }^{1,16,21,28}$ Although spontaneous herniation is now being recognized more frequently on MR imaging, posttraumatic herniation remains a rare condition with only a small number of reports in the literature. ${ }^{6-9,14,15,19,22,25}$ In some of these posttraumatic cases, the presumed cause has been based on a history of remote and sometimes trivial trauma, which, in our opinion, raises the possibility that these cases are not related to injury but are more likely idiopathic (spontaneous) in occurrence. The usual clinical presentation of spontaneous spinal cord herniation is that of myelopathy and Brown-Séquard syndrome affecting middle-age patients. There is a female/male ratio of approximately $2: 1.4,12,13,16-18,25,28$ Cases of isolated motor and sensorimotor deficits, impotence, and bowel and bladder dysfunction affecting younger patients, on the other hand, have been observed after traumatic injury or surgery..$^{25,28}$ Spontaneous spinal cord herniation is often thoracic in location and the spinal cord is displaced ventrally. ${ }^{12,17,20,23}$ Posttraumatic and iatrogenic herniation occurs throughout the spine, with cases of cervical posterolateral herniation developing after $\mathrm{C} 1-2$ wiring failure and direct posterior conus medullaris prolapse after L-1 burst fracture. ${ }^{28}$

In cases of traumatic spinal cord herniation, patients usually present after a postinjury interval of several years, rarely within few days of the initial injury. For example, in 1997 Lee, et al., ${ }^{14}$ reported on a case of delayed paraplegia associated with spinal cord herniation 2 days after a stab

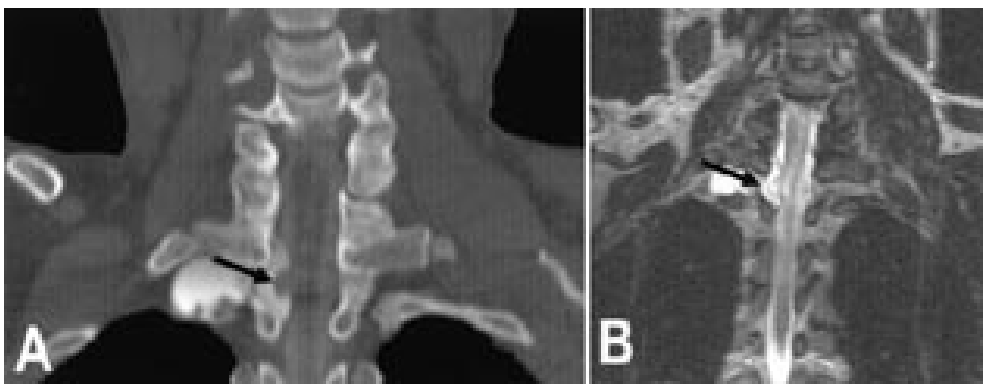

FIG. 2. Coronal postmyelography CT scan (A) and fast-spin echo $\mathrm{T}_{2}$-weighted MR image (B) revealing an intra- and extraspinal pseudomeningocele and dural hiatus with spinal cord herniation (arrow). 


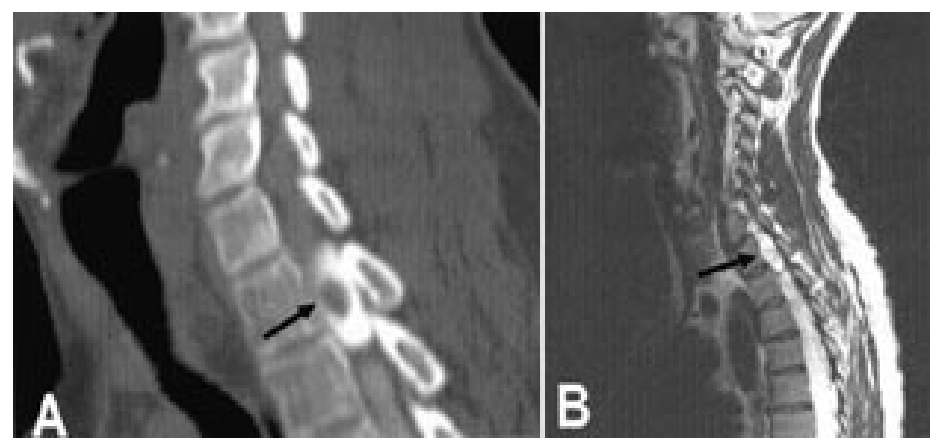

FIG. 3. Sagittal postmyelography CT scan (A) and fast-spin echo $\mathrm{T}_{2}$-weighted MR image (B) demonstrating an intraspinal cyst with the herniated spinal cord (arrow).

injury to the spine, whereas in 1996 Urbach, et al., ${ }^{25}$ described a case of thoracic spinal cord herniation 38 years after the patient suffered blunt trauma to the spine (Table 1).

In 1995 Kumar, et al. ${ }^{13}$ proposed that a dural defect located at the concavity of the spinal curvature, development of arachnoidal adhesions, and CSF pulsation-induced anterior-posterior movement of the spinal cord are pivotal factors leading to the herniation of the cord out of its dural sleeve into a preexisting cyst. ${ }^{13}$ Some authors have theorized that a herniated, calcified disc as well as intradural disc herniation can erode the dura mater; such theories that have been proposed to explain the dural hiatus in spontaneous spinal cord herniation, which seems to be of degenerative rather than congenital origin because of the characteristic time of presentation. ${ }^{10,16,17,29}$

Radiologically, spinal cord kinking and neural tissue prolapse are the hallmarks of this disorder. ${ }^{10,18,24,28}$ Spinal cord atrophy, intramedullary hyperintensity, syrinx formation, tethering, vertebral body nuclear trail sign, and scalloping of vertebral body have been also observed. ${ }^{2,16,28}$ Postmyelography CT scanning and phase-contrast MR imaging are helpful in demonstrating the normal CSF flow pattern dorsally and excluding the possibility of a compressive posterior arachnoidal cyst. ${ }^{5}$ Intraoperatively, herniation of the spinal cord and nerve roots through a dural defect or duplicated dura have been reported. ${ }^{1,12,17,18,20}$

In the present case, one can speculate that traumatic avulsion of the T-1 nerve root caused a dural "hiatus" at the origin of the nerve root sleeve, through which CSF extravasated and created the observed complex pseudomeningocele; here CSF pulsations ultimately culminated in the herniated spinal cord. Arachnoidal adhesions likely developed over time, leading to tethering of the cord to the dural ring and thereby preventing the cord from returning into the spinal canal.

The rarity of this condition makes it hard to define a treatment algorithm. It is, however, prudent to follow expectant therapy in asymptomatic cases. This also seems reasonable for mildly symptomatic and nonprogressive cases. ${ }^{16}$ In more severe and progressive cases, surgical repair is the intervention of choice. This can be achieved via primary closure with or without placement of a dural patch, using muscle, fascia graft, synthetic membrane, or lyophilized dura ${ }^{4,17,20,29}$ or, less commonly, by enlarging the dural opening and untethering the spinal cord alone. ${ }^{27}$
Despite the retrospective nature of the vast majority of reported cases, outcome appears favorable in most surgically treated patients, with persistent sensory symptoms

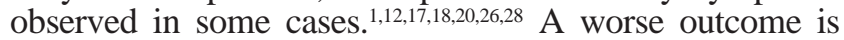
associated with an inability to reduce fully the herniated spinal cord because of the presence of extensive arachnoidal adhesions limiting untethering of the spinal cord. ${ }^{3,11}$ Increasing awareness of this disorder seems to be the key to establishing early diagnosis and preserving functional status in patients presenting with myelopathy or BrownSéquard syndrome but in whom there is no evidence of extrinsic spinal cord compression.

\section{Conclusions}

Spinal cord herniation following traumatic nerve root avulsion is rare but should be considered in patients presenting with delayed Brown-Séquard syndrome or myelopathy. Appropriate MR imaging allows exclusion of compressive disease such as disc, tumor, or pseudomeningocele, and specific diagnosis. Surgical repair is recommended for symptomatic cases; this intervention is technically feasible and leads to improvement or at least stabilization of the neurological status.

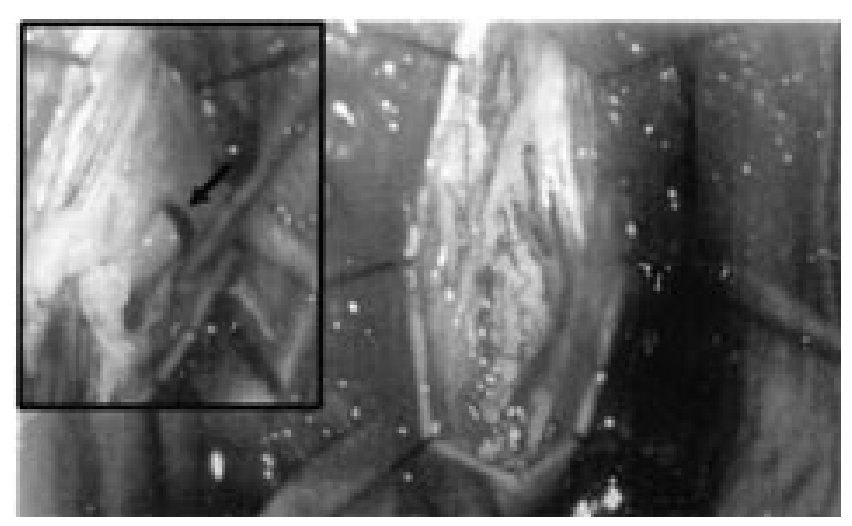

FIG. 4. Intraoperative photographs illustrating the spinal cord herniating through the intradural defect. Inset: the dural defect is shown after micolysis of adhesions and reduction of the spinal cord hernia. 
TABLE 1

Summary of reported cases of posttraumatic spinal cord herniation*

\begin{tabular}{|c|c|c|c|c|c|c|c|}
\hline Authors \& Year & $\begin{array}{c}\text { Age (yrs), } \\
\text { Sex }\end{array}$ & $\begin{array}{l}\text { Spinal } \\
\text { Level }\end{array}$ & $\begin{array}{c}\text { Herniation } \\
\text { Direction }\end{array}$ & $\begin{array}{l}\text { Spinal } \\
\text { Pain }\end{array}$ & Clinical Deficits & $\begin{array}{l}\text { Yrs Since } \\
\text { Trauma }\end{array}$ & Postop Course \\
\hline Wortzman, et al., 1974 & $63, \mathrm{M}$ & $\mathrm{T}-7$ & anterior & yes & myelopathy & 31 & worsened, then improved \\
\hline Sachdev, et al., 1981 & $44, \mathrm{~F}$ & L-1 & rt anterior & yes & rt leg sensorimotor & 6 & worsened, then improved \\
\hline Borges, et al., 1995 & $68, \mathrm{M}$ & $\mathrm{T} 2-3$ & lt anterior & no & Brown-Séquard & 48 & improved \\
\hline Urbach, et al., 1996 & $44, \mathrm{M}$ & T5-6 & rt anterior & no & myelopathy & 38 & improved \\
\hline \multirow[t]{3}{*}{ Watters, et al., 1998} & $46, \mathrm{M}$ & T-6 & lt anterior & yes & lt leg pyramidal & 3 & improved, then worsened \\
\hline & $35, \mathrm{M}$ & $\mathrm{T}-3$ & posterior & NR & myelopathy & 17 & unknown \\
\hline & $46, \mathrm{M}$ & L-1 & rt posterior & yes & myelopathy & 1 & unchanged \\
\hline present case & $41, \mathrm{M}$ & $\mathrm{T}-1$ & rt lat & no & myelopathy & 19 & early improvement \\
\hline
\end{tabular}

$* \mathrm{NR}=$ not reported.

\section{Acknowledgment}

We are grateful to Ms. Catherine Munro for her assistance with preparing the figures and the manuscript.

\section{References}

1. Aizawa T, Sato T, Tanaka Y, et al: Idiopathic herniation of the thoracic spinal cord: report of three cases. Spine 26: E488-E491, 2001

2. Awwad EE, Martin DS, Smith KR Jr: The nuclear trial sign in thoracic herniated disks. AJNR Am J Neuroradiol 13:137-143, 1992

3. Berbel A, Porta-Etessam J, Martinez-Salio A, et al: [Idiopathic spinal cord herniation. Presentation of a new case and review of the literature.] Rev Neurol 32:54-57, 2001 (Sp)

4. Borges LF, Zervas NT, Lehrich JR: Idiopathic spinal cord herniation: a treatable cause of the Brown-Sequard syndrome-case report. Neurosurgery 36:1028-1033, 1995

5. Brugieres $\mathrm{P}$, Malapert D, Adle-Biassette H, et al: Idiopathic spinal cord herniation: value of MR phase-contrast imaging. AJNR Am J Neuroradiol 20:935-939, 1999

6. Burres KP, Conley FK: Progressive neurological dysfunction secondary to postoperative cervical pseudomeningocele in a C-4 quadriplegic. Case report. J Neurosurg 48:289-291, 1978

7. Cobb C III, Ehni G: Herniation of the spinal cord into an iatrogenic meningocele. Case report. J Neurosurg 39:533-536, 1973

8. Dunn V, Smoker WR, Menezes AH: Transdural herniation of the cervical spinal cord as a complication of a broken fracturefixation wire. AJNR Am J Neuroradiol 8:724-726, 1987

9. Goodman SJ, Gregorius FK: Cervical pseudomeningocele after laminectomy as a cause of progressive myelopathy. Bull Los Angeles Neurol Soc 39:121-127, 1974

10. Hausmann ON, Moseley IF: Idiopathic dural herniation of the thoracic spinal cord. Neuroradiology 38:503-510, 1996

11. Henry A, Tunkel R, Arbit E, et al: Tethered thoracic cord resulting from spinal cord herniation. Arch Phys Med Rehabil 78: 530-533, 1997

12. Isu T, Iizuka T, Iwasaki Y, et al: Spinal cord herniation associated with an intradural spinal arachnoid cyst diagnosed by magnetic resonance imaging. Neurosurgery 29:137-139, 1991

13. Kumar R, Taha J, Greiner AL: Herniation of the spinal cord. Case report. J Neurosurg 82:131-136, 1995

14. Lee ST, Lui TN, Jeng CM: Spinal cord herniation after stabbing injury. Br J Neurosurg 11:84-86, 1997

15. Marquardt G, Weidauer S, Zanella FE, et al: Acute posttraumatic spinal cord herniation. Case report and review of the literature. J Neurosurg (Spine 2) 94:316-318, 2001

16. Massicotte EM, Montanera W, Ross Fleming JF, et al: Idiopathic spinal cord herniation: report of eight cases and review of the literature. Spine 27:E233-E241, 2002
17. Masuzawa H, Nakayama H, Shitara N, et al: Spinal cord herniation into a congenital extradural arachnoid cyst causing BrownSéquard syndrome. Case report. J Neurosurg 55:983-986, 1981

18. Miura Y, Mimatsu K, Matsuyama Y, et al: Idiopathic spinal cord herniation. Neuroradiology 38:155-156, 1996

19. Mizuno J, Nakagawa H, Iwata K: [Postoperative spinal cord herniation diagnosed by metrizamide CT: a case report.] No Shinkei Geka 14:681-685, 1986 (Jpn)

20. Nakazawa H, Toyama Y, Satomi K, et al: Idiopathic spinal cord herniation. Report of two cases and review of the literature. Spine 18:2138-2141, 1993

21. Pereira P, Duarte F, Lamas R, et al: Idiopathic spinal cord herniation: case report and literature review. Acta Neurochir (Wien) 143:401-406, 2001

22. Sachdev VP, Huang YP, Shah CP, et al: Posttraumatic pseudomeningomyelocele (enlarging fracture?) in a vertebral body. Case report. J Neurosurg 54:545-549, 1981

23. Tronnier VM, Steinmetz A, Albert FK, et al: Hernia of the spinal cord: case report and review of the literature. Neurosurgery 29:916-919, 1991

24. Uchino A, Kato A, Momozaki N, et al: Spinal cord herniation: report of two cases and review of the literature. Eur Radiol 7: 289-292, 1997

25. Urbach H, Kaden B, Pechstein U, et al: Herniation of the spinal cord 38 years after childhood trauma. Neuroradiology 38: $157-158,1996$

26. Vallee B, Mercier P, Menei P, et al: Ventral transdural herniation of the thoracic spinal cord: surgical treatment in four cases and review of literature. Acta Neurochir (Wien) 141:907-913, 1999

27. Watanabe M, Chiba K, Matsumoto M, et al: Surgical management of idiopathic spinal cord herniation: a review of nine cases treated by the enlargement of the dural defect. J Neurosurg (Spine 2) 95:169-172, 2001

28. Watters MR, Stears JC, Osborn AG, et al: Transdural spinal cord herniation: imaging and clinical spectra. AJNR Am J Neuroradiol 19:1337-1344, 1998

29. Wortzman G, Tasker RR, Rewcastle NB, et al: Spontaneous incarcerated herniation of the spinal cord into a vertebral body: a unique cause of paraplegia. Case report. J Neurosurg 41: 631-635, 1974

Manuscript received January 21, 2003

Accepted in final form June 16, 2003.

Address reprint requests to: Rajiv Midha, M.D., Division of Neurosurgery, Department of Surgery, Sunnybrook and Women's College Health Sciences Centre, Suite A131, 2075 Bayview Avenue, Toronto, ON, Canada M4N 3M5. email: rajiv.midha@sw.ca. 